\title{
Phase diagram of force-induced DNA unzipping in exactly solvable models
}

\author{
D. Marenduzzo ${ }^{1, *}$, A. Trovato ${ }^{2}$, and A. Maritan ${ }^{1}$ \\ ${ }^{1}$ International School for Advanced Studies (SISSA), \\ and Istituto Nazionale di Fisica della Materia, \\ Via Beirut 2-4, 34014 Trieste, Italy \\ ${ }^{1}$ The Abdus Salam International Center for Theoretical Physics, \\ Strada Costiera 11, 34100 Trieste, Italy \\ ${ }^{2}$ Niels Bohr Institutet, Blegdamsvej 17, 2100 København Ø, Denmark
}

\begin{abstract}
The mechanical separation of the double helical DNA structure induced by forces pulling apart the two DNA strands ("unzipping") has been the subject of recent experiments. Analytical results are obtained within various models of interacting pairs of directed walks in the $(1,1, \ldots, 1)$ direction on the hypercubic lattice, and the phase diagram in the force-temperature plane is studied for a variety of cases. The scaling behaviour is determined at both the unzipping and the melting transition. We confirm the existence of a cold denaturation transition recently observed in numerical simulations: for a finite range of forces the system gets unzipped by decreasing the temperature. The existence of this transition is rigorously established for generic lattice and continuum space models.
\end{abstract}

\section{INTRODUCTION}

In recent years, micro and nanomanipulation of single biological macromolecules has become feasible due to a dramatic improvement of experimental techniques. By using devices such as optical tweezers [1.2], soft microneedles [3] and atomic force microscopes [4], it is now possible to access physical and mechanical properties of fundamental biological objects, namely proteins, nucleic acids, and molecular motors, on the scale of individual molecules. Special effort has been devoted to the measurement of force-elongation characteristics of double stranded DNA molecules (dsDNA), determining its response to external forces and torques in the absence of enzymes. The mechanical unzipping of dsDNA structure by a force pulling the end of one of the two strands, the end of the other strand being anchored to some physical support, has been studied by Bockelmann et al. [5.,6], who have measured the average force along the opening of the two strands. Mechanical forces are in fact exerted on the DNA molecule by different enzymes during the process of DNA replication or transcription [7]:8]. On the other hand, the double helical structure of dsDNA may be disrupted 'in vitro' by changing $\mathrm{pH}$, solvent conditions and/or temperature [9]. This transition is known as melting denaturation, and it has been long studied by theoretical physicists [10 12]. Instead, only very recently DNA mechanical denaturation has been the subject of theoretical studies. Most of these studies have considered so far a simple extension of the Poland-Sheraga model [11, in which the two DNA strands are homogeneous ideal polymer chains interacting with each other, introducing a constant force pulling apart the two strands by acting on one extremity of both strands. By using a mapping into a quantum mechanical problem, it has been shown [13 16] that the opening of the two strands occurs only if the pulling force is increased to a critical value. The unzipping transition turns out to be a first order phase transition, whereas the melting transition is second order, in the ideal case, in $d=3$ (recently both simulations and exact results have shown that the melting transition becomes first order, when considering mutually self-avoiding walks (SAWs) [17, 18]). The heterogeneous case has also been studied with similar techniques [14]. Very recently, Monte Carlo simulations of interacting pairs of self avoiding walks on a cubic lattice and of bead-and-string chains in the continuum space have determined the whole phase diagram in the force-temperature plane, revealing the existence of a re-entrant zipping-unzipping transition by decreasing the temperature for a finite range of forces 19 .

In this paper, we obtain exact analytical results for a class of simple lattice models of interacting pairs of homogeneous directed self-avoiding walks. We extend the model introduced in ref. $[19 \|$ in $D=1+1$ to the generic dimension case $D=d+1$, and analyze both the scaling behavior of the first order unzipping transition and the multicritical scaling laws at the melting transition in the force-temperature plane. We also consider different versions of the model depending on whether the crossing of the two walks is allowed or not, or simply penalized by an entropy cost. In all cases, we find that the critical pulling force increases with temperature at low $T$, implying thus the existence of a cold unzipping transition [19]. This seemingly paradoxical property is due to the competition between the energy gained by increasing the open portion of DNA and the entropy lost with the full stretching of the separated strands. Cold unzipping will be exactly proved for both ideal and self-avoiding chains in the lattice, and for a discrete chain with constant distance between consecutive beads in the continuum space. We also discuss the role of denatured bubbles forming in dsDNA opening, as opposed to the end-opening of the strands induced by the pulling force. By comparing 
the phase diagram of the different models considered, we point out that the approximation of neglecting bubbles and considering only Y-shaped configurations shares many of the features of a mean-field type approximation, with an upper critical dimension $d_{c}=4$.

Our paper is organized as follows. In Sec.II we introduce the models of directed walks on the lattice in any dimension $D$ and compute their thermodynamical properties, deriving the phase diagram in the force-temperature plane. In Sec.III we analyze the scaling laws at both the thermal melting and mechanical unzipping transition, and we highlight the features that are physically more interesting in our exact results (namely the existence of a re-entrant cold unzipping transition, our main result, and the role of forbidding the mutual crossing of the two strands). In Sec.IVa we discuss the physical meaning of re-entrance in our (lattice) models, generalizing the proof of its occurrence to the more realistic case of SAWs. In Sec.IVb we prove that also a discrete chain in the continuum space undergoes cold unzipping, and then argue why such an effect had not been observed in previous calculations [14 16], performed in the limit of a continuum chain. Eventually, we discuss some related models (with generic SAWs) and give some additional technical details in the Appendices.

\section{EXACT SOLUTION OF THE LATTICE MODELS}

\section{A. Introduction of the models and their behaviour for thermal melting}

We consider a simple class of models in a $D=d+1$ dimensional hypercubic lattice $\mathbf{Z}^{\mathbf{D}}$. The two strands of a homogeneous DNA molecule with $N$ base pairs are mimicked by two SAWs, directed along the $(1, \ldots, 1)$ direction. The two chains have one end in common, while at the other end a force $\vec{g}$ is pulling in the $(1,-1,0, \ldots, 0)$ direction. The walks gain a binding energy $-\epsilon(\epsilon>0)$ every time the bases with the same monomer index (or same projection upon $(1, \ldots, 1))$ interact, i.e. wrong base pairing is forbidden.

The canonical partition function for two $N$-step directed self avoiding walks (DSAWs) is:

$$
Z_{N}(\beta, \vec{g})=\sum_{\vec{x} \in \mathbf{Z}^{\mathrm{D}}} p_{N}(\vec{x}, \beta \epsilon) \exp (\beta \vec{g} \cdot \vec{x}),
$$

where $p_{N}(\vec{x}, \beta \epsilon)$ is the canonical partition function of two directed interacting strands whose last base pairs are at relative distance $\vec{x}$. The following recursion relation holds for $p_{N}(\vec{x}, \beta \epsilon)$ :

$$
p_{N+1}(\vec{x}, \beta \epsilon)=\sum_{i, j=1}^{D} p_{N}\left(\vec{x}-\vec{e}_{i}+\vec{e}_{j}, \beta \epsilon\right)\left(1+(\exp (\beta \epsilon)-1) \delta_{\vec{x}, \overrightarrow{0}}\right),
$$

where $\vec{e}_{i}, i=1, \ldots, D$, are the canonical euclidean versors of the $D$-dimensional space and $\delta$ is the Kronecker delta.

These and similar equations have been intensively studied, at $\vec{g}=\overrightarrow{0}$, within simple models of DNA thermal melting [17, 19], and, in $D=2$, within the context of random walk adsorption 20 and wetting problems [21]. Note that the choice of the $(1, \ldots, 1)$ direction along which the walks are directed, is crucial in allowing us to write local recursion relations. It is thus no surprise that the model belongs to the same universality class of random walks in $d=D-1$ dimensions [22].

It is well known (see 17 for a recent review), that, in the absence of a pulling force, within the model defined by equation (2), dsDNA undergoes a phase transition between a low temperature double stranded phase and a high temperature denaturated state only for $D>3(d>2)$ : in $D=2,3$ dsDNA remains double stranded at all temperatures.

However, we note that in (2) the two strands are allowed to cross each other, without any restriction. This seems rather unphysical, since every real chain has a finite "hard core" distance. Thus, one should consider also the case in which crossing between different strands is forbidden (recent studies of both homogeneous [23] and heterogeneous 24. DNA melting transition have indeed considered the $d=1$ case with forbidden crossing). Let us focus for the moment on the $D=2$ case, with no pulling force, where the effect of forbidding crossing is most dramatic. Here, the direction $(1 / \sqrt{2}, 1 / \sqrt{2})$ can be identified as "time", and its normal $(1 / \sqrt{2},-1 / \sqrt{2})$ as "space" $(x)$. The model with crossing (w.c.) implies no restriction on the relative distance $x$, whereas the one without crossing (w.o.c.) implies that $x$ cannot change sign, e.g. $x \geq 0$. The model w.o.c. is equivalent to surface adsorption models previously considered [20]. While the thermal melting of the two strands w.c. takes place at $T_{c}=\infty$, in the model w.o.c. $T_{c}=\epsilon / \log (4 / 3)$ as it can be deduced from ref. 20]. To elucidate this point further, we have tackled an intermediate model, where we do not forbid crossing, but we make it disadvantageous, by assigning a cost $V>0$ each time the strands pass 
through one another. With calculations similar to those reported below, we find that melting takes place at the critical temperature (as a function of $V$ ):

$$
T_{c}(V)=\epsilon\left[\log \left(\frac{4 e^{V}}{3 e^{V}+1}\right)\right]^{-1} .
$$

As expected, as $V \rightarrow \infty$, equation (3) yields $T_{c} \rightarrow \epsilon / \log (4 / 3)$, whereas $T_{c} \sim \epsilon / V$ as $V \rightarrow 0$.

To implement the non-crossing constraint in the model in generic dimension, we require that, if the DSAWs join, one coming from direction $\vec{e}_{i}$ and the other from direction $\vec{e}_{j}$ (with $i \neq j$ ), when they divide again, they cannot both proceed along their previous direction; i.e we forbid that the first walk goes along direction $\vec{e}_{i}$ and the second one along $\vec{e}_{j}$. This excludes one configuration out of $D(D-1)$ at the splitting point (the remaining $D$ possibilities would lead to the zipping of the two strands). As regards the $D=3$ thermal melting, again the model w.c. has $T_{c}=\infty$, while, if crossing is forbidden as described above, the critical temperature is $T_{c}=\epsilon / \log (9 / 8)$. This result was also found in a similar calculation by Rubin [25]. For $D>3$ the models with and without crossing both undergo a denaturation transition at a finite, though different, critical temperature, so that the effect of forbidding crossing here is less important (the critical temperatures are the same at the leading order $1 / D$ in the $D \rightarrow \infty$ expansion, as expected).

\section{B. Behaviour of the models at non zero force}

Let us now turn to the models with the pulling force $\vec{g}$ in generic dimension $D$, with $\vec{g}=(g,-g, 0, \ldots, 0)$. We can find the asymptotic value of the canonical partition function (1) by locating [17, 19,26] the singularity closest to the origin of its generating function:

$$
Z(z, \beta \epsilon, \beta g)=\sum_{N=0}^{\infty} z^{N} \sum_{\vec{x}} p_{N}(\vec{x}, \beta \epsilon) \exp (\beta \vec{g} \cdot \vec{x})
$$

which we will also refer to as the grand partition function. We stress that $p_{N}(\vec{x}, \beta \epsilon)$ is a generic partition function: its detailed form will depend on whether or not we allow crossing.

To proceed, it is useful to partition the DNA molecule in ds helices (with the strands attached to each other) and bubbles, sequences in which the two chains share just the first and the last base pairs; we also single out the contribution of the unzipped end of the two DSAWs, i.e. the part from the last contact to the end. In this way, the grand partition sum (4) can be expressed as:

$$
Z(z, \beta \epsilon, \beta g)=\frac{1}{1-D z \exp (\beta \epsilon)} \frac{\exp (\beta \epsilon)}{1-\frac{\exp (\beta \epsilon)}{1-D z \exp (\beta \epsilon)} B(z)} S(z, \beta g)
$$

where we have defined:

$$
B(z)=\sum_{N=2}^{\infty} z^{N} b_{N}, \quad S(z, \beta g)=1+\sum_{N=1}^{\infty} z^{N} \sum_{\vec{x}}\left[c_{N-1}(\vec{x})-c_{N-1}(\overrightarrow{0}) \delta_{\vec{x}, \overrightarrow{0}}\right] \exp (\beta \vec{g} \cdot \vec{x}) .
$$

In (6),$b_{N}$ is the number of $2 N$-step bubbles, and $c_{N}(\vec{x})$ the partition function of two $N$-step DSAWs never touching each other and having their last sites at a mutual distance $\vec{x}$, and their initial sites at a relative distance $\vec{e}_{i_{0}}-\vec{e}_{j_{0}}$ for some $i_{0} \neq j_{0}$. By summing over all possibilities for initial conditions (see equation (8) below), $b_{N}=\sum_{i \neq j ; i, j=1}^{D} c_{N-2}\left(\vec{e}_{i}-\vec{e}_{j}\right.$ ), so that we need to find an explicit expression for the $c_{N}$ 's. The equations they obey are:

$$
c_{N+1}(\vec{x})=\sum_{i, j=1}^{D} c_{N}\left(\vec{x}-\vec{e}_{i}+\vec{e}_{j}\right)-c_{N}(\overrightarrow{0}) \sum_{i \neq j ; i, j=1}^{D} \delta_{\vec{x}, \vec{e}_{i}-\vec{e}_{j}}
$$

Notice that in eq. (17) $\overrightarrow{0}$ acts as a sink or absorbing state, i.e. once the two walks join, they never leave. In this way, $c_{N}(\vec{x})$ with $\vec{x} \neq \overrightarrow{0}$ counts the number of pairs of walks that never touch each other (and with the last monomers at a relative distance $\vec{x})$, while $c_{N}(\overrightarrow{0})$ counts the number of remaining pairs of walks that at some point come into contact and then remain stuck together. This last quantity plays the role of an arbitrary constant and has to be subtracted away from the final counting as in (6) (see 25 for another example in which this prescription was used in a $D=3$ example). 
In order to simplify the presentation from now on we restrict our calculations on models w.o.c. (detailed calulations for this case and the outline of calculations for the simpler models w.c. are deferred to Appendix A). The initial conditions for such models are:

$$
c_{0}(\vec{x})=\left(\sum_{i \neq j ; i, j=1}^{D} \delta_{\vec{x}, \vec{e}_{i}-\vec{e}_{j}}\right)-\delta_{\vec{x}, \vec{e}_{i_{0}}-\vec{e}_{j_{0}}},
$$

where $\vec{e}_{i_{0}}$ and $\vec{e}_{j_{0}}$ are the directions forbidden as described in the previous subsection.

By performing a Fourier and a discrete Laplace transform on eq.(7), and by using the relation between $b_{N}$ and $c_{N}$ given above, we can derive an explicit expression for the bubble generating function $B(z)$ (see eq. (A6 $)$ ). The singularities of the second denominator of the grand partition function (5) are necessary to find the melting temperature. We find that the equations locating these two singularities are :

$$
\begin{aligned}
z_{1} & =\frac{1}{D^{2}}, \\
\exp (-\beta \epsilon)-D z_{2} & =B\left(z_{2}\right) .
\end{aligned}
$$

The first singularity $z_{1}$ is leading to the usual random walk behavior in the absence of any interaction. The second singularity $z_{2}(\beta \epsilon)$ is a function of the strength of the attractive interaction between the two strands, determining thus the behaviour in the native zipped phase. At $g=0$, the critical temperature for thermal melting is obtained when the two singularities above coincide $\left(z_{1}=z_{2}\right)$. Unlike the models w.c., which have $T_{c}=\infty$ in $D \leq 3$, those w.o.c. have a finite critical temperature in any $D \geq 2$ (see Appendix A for further details).

As regards the force-dependent third factor in (5), it reads:

$$
S(z, \beta g)=z S_{\text {sing }}(z, \beta g)+1-z c(z, \overrightarrow{0}) .
$$

A third new singularity, depending on the external force, and fundamental in our calculations arises when computing:

$$
S_{\text {sing }}(z, \beta g) \equiv \int_{[-\pi, \pi]^{D}} \frac{d^{D} \vec{q}}{(2 \pi)^{D}} \widetilde{c}(z, \vec{q}) \sum_{\vec{x}} \exp [(\beta \vec{g}-i \vec{q}) \cdot \vec{x}]
$$

where $\tilde{c}(z, \vec{q})$ and $c(z, \overrightarrow{0})$ are given in eqs. (A4, A5). Notice that in eq. (12) one can immediately compute the integrals on $d q_{3} \ldots d q_{D}$, by using the well known identity $2 \pi \delta(q)=\sum_{x} \exp (-i q x)$, so that one is left with the double integral:

$$
\int_{-\pi}^{\pi} \frac{d q_{2}}{(2 \pi)} \int_{-\pi}^{\pi} \frac{d q_{1}}{(2 \pi)} \tilde{c}\left(z, q_{1}, q_{2}, 0, \ldots, 0\right) \sum_{x_{1}, x_{2}} \exp \left[\left(\beta g-i q_{1}\right) x_{1}+\left(-\beta g-i q_{2}\right) x_{2}\right]
$$

We now give details for the evaluation of the inner integral only, the outer one being equivalent. Defining $h\left(q_{1}, q_{2}\right) \equiv$ $\left(\tilde{c}\left(z, q_{1}, q_{2}, 0, \ldots, 0\right)\right)^{-1}$, we aim at proving that the complex translation $q_{1} \rightarrow q_{1}-i \beta g$ can be performed in the integral $\int_{-\pi}^{\pi} \frac{d q_{1}}{2 \pi} \frac{\exp \left(-i q_{1} x_{1}\right)}{h\left(q_{1}, q_{2}\right)}$. Notice that $h$ is a periodic function of $q_{1}$. We extend $q_{1}$ to complex numbers and consider the contour $\gamma$ as shown in Fig. 1. By the residue theorem, one can write:

$$
\begin{gathered}
\int_{-\pi}^{\pi} \frac{d q_{1}}{2 \pi} \frac{\exp \left(-i q_{1} x_{1}\right)}{h\left(q_{1}, q_{2}\right)}+\int_{0}^{-\beta g} \frac{i d y}{2 \pi} \frac{\exp \left(-i \pi x_{1}+x_{1} y\right)}{h\left(\pi+i y, q_{2}\right)}- \\
\int_{-\pi}^{\pi} \frac{d q_{1}}{2 \pi} \frac{\exp \left(-i q_{1} x_{1}-\beta g x_{1}\right)}{h\left(q_{1}-i \beta g, q_{2}\right)}+\int_{-\beta g}^{0} \frac{i d y}{2 \pi} \frac{\exp \left(i \pi x_{1}+x_{1} y\right)}{h\left(-\pi+i y, q_{2}\right)}=\sum_{q_{0}} \operatorname{Res}\left(\mathcal{H}, q_{0}\right),
\end{gathered}
$$

where the sum runs over all poles $q_{0}$ of $\mathcal{H} \equiv \frac{\exp \left(-i q_{1} x_{1}\right)}{h\left(q_{1}, q_{2}\right)}$ (at fixed $\left.q_{2}\right)$ inside the contour $\gamma$ and $\operatorname{Res}\left(\mathcal{H}, q_{0}\right)$ is the residue of $\mathcal{H}$ at $q_{0}$. Note that due to the periodicity of $h$ the second and fourth terms in the previous equation cancel and thus the complex translation can be performed provided that no pole of $\mathcal{H}$ can be found inside the contour $\gamma$. An equivalent conclusion can be reached for the outer integral on $d q_{2}$. The condition of having no poles inside the contours of integration is satisfied as long as $z<z_{3}(\beta g)$, where:

$$
z_{3}(\beta g)=\frac{1}{(D-2)^{2}+2+2 \cosh (2 \beta g)+4(D-2) \cosh (\beta g)}
$$


is found by solving $h(-i \beta g, i \beta g) \propto(1-z f(-i \beta \vec{g}))=0$, with $f(\vec{q})$ defined in eq. (A2). In this case, we find consistently that $z_{3}(\beta g)$ is just the singularity in the resulting expression $S(z, \beta g)=1+z[\tilde{c}(z,-i \beta \vec{g})-c(z, \overrightarrow{0})]$.

As $z_{3}$ is always smaller than $z_{1}$ (for $g \neq 0$ ), the singularity closest to the origin has to be determined between $z_{2}$, controlled by the attractive energy $\epsilon$, and $z_{3}$, controlled by the pulling force $g$. If $z_{2}<z_{3}$, the DNA molecule is zipped, otherwise it is unzipped. The free energy per monomer $f$ and the average distance between the two ends (projected onto $\hat{g} \equiv(1,-1,0, \ldots, 0)),\left\langle x_{g}>\right.$, are defined as:

$$
\begin{aligned}
& f \equiv \lim _{N \rightarrow \infty}-T \frac{\log \left[\sum_{\vec{x}} p_{N}(\vec{x}, \beta \epsilon) \exp (\beta \vec{g} \cdot \vec{x})\right]}{N} \\
& <x_{g}>\equiv \lim _{N \rightarrow \infty}<\hat{g} \cdot \vec{x}>=-\frac{\partial f}{\partial g} N
\end{aligned}
$$

These quantities read in the thermodynamic limit:

$$
\begin{aligned}
& f=T \log z_{2}(\beta \epsilon) ; \frac{<x_{g}>}{N}=0 \quad g<g_{c}(T, \epsilon) \\
& f=T \log z_{3}(\beta g) ; \frac{<x_{g}>}{N}=z_{3}(\beta g)[4 \sinh (2 \beta g)+4(D-2) \sinh (\beta g)] \quad g>g_{c}(T, \epsilon)
\end{aligned}
$$

where the critical force $g_{c}(T, \epsilon)$ is found by imposing $z_{2}(\beta \epsilon)=z_{3}(\beta g)$, as given in eqs. 10,15$)$. The above equations show the existence of a first order transition at $g=g_{c}(T, \epsilon)$, if $g_{c}(T, \epsilon)>0$. The phase diagram for the models w.c. can be found exactly in the same way (see Appendix A for some details on this). It is interesting to notice that the singularity $z_{3}(\beta g)$ does not depend on whether or not we allow crossing, whereas $z_{2}$ in the w.c. case is different from the w.o.c. case. This is enough of course to make the whole phase diagram different for the two kinds of models, as will be discussed in section III.

We stress here that the main interest in using directed walks is that they are a sub-class of SAWs in the same dimension. However, qualitatively similar results can be obtained also with simple random walks (RWs) [28], but in that case it would be not physically meaningful to forbid crossing as done above.

Experimentally, however, it should not be hard to set up an experiment closely related to the calculation we have performed, by stretching the ds molecule in one given direction before applying the external force.

\section{SCALING LAWS FOR THERMAL MELTING AND UNZIPPING}

Let us focus for concreteness on the result for the model in $D=2$ w.o.c., in order to analyze the scaling laws of the system. The phase diagram, as found also in [19], explicitly reads (see Fig. 2):

$$
g_{c}(T, \epsilon)=\frac{T}{2} \cosh ^{-1}\left[\frac{1}{2} \frac{1}{\sqrt{1-\exp (-\beta \epsilon)}-(1-\exp (-\beta \epsilon))}-1\right] .
$$

The model exhibits a first-order "unzipping" transition if we move at a fixed value of $T<\epsilon / \log (4 / 3)$, as shown in (18). As is shown in Fig. 2, $\lim _{T \rightarrow 0} g_{c}(T, \epsilon)=\frac{\epsilon}{2}$ and $g_{c}(T, \epsilon)$ attains its maximum at $T=T_{M} \simeq 0.9 \epsilon$ where $g_{c}\left(\overline{T_{M}}\right) \simeq 0.68 \epsilon>\frac{\epsilon}{2}$. The transition is second order at $g=0$; as in [17] we find that close to the melting point

$$
<n>\equiv \frac{\partial}{\partial(\beta \epsilon)} \log Z_{N} \sim \tau^{-1} f\left(\tau N^{\phi_{t}}\right),
$$

where $\langle n\rangle$ is the mean number of native contacts, with $\tau=\left(T-T_{c}\right) / T_{c}$, and $\phi_{t}=1 / 2$ for the crossover exponent at thermal melting in the $D=2$-case. The scaling function $f(x)$ behaves as usual

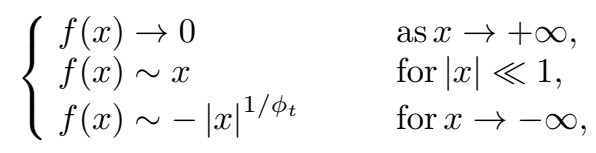

such that $<n>\sim N^{\phi_{t}}$ at the transition.

The exact expressions for $\langle n\rangle$ and for $\left\langle x_{g}\right\rangle$ can be found by inverse Laplace transforms of the quantities $Z(z, \beta g, \beta \epsilon), \frac{\partial}{\partial(\beta \epsilon)} Z(z, \beta g, \beta \epsilon), \frac{\partial}{\partial(\beta g)} Z(z, \beta g, \beta \epsilon)$. However, if we only need scaling relations in the thermodynamic limit, we can use the discrete Tauberian theorem (see [27] and Appendix B), which relates the critical behaviour of a 
series to the asymptotic behaviour of its coefficients. By using this method, we find, in the vicinity of the unzipping mechanical transition:

$$
\begin{aligned}
& <n>\sim \frac{h_{1}\left[N^{\phi_{1}}\left(A_{1} \gamma+A_{2} \tau\right)\right]}{A_{1} \gamma+A_{2} \tau}, \\
& <x_{g}>\sim-\frac{h_{2}\left[-N^{\phi_{2}}\left(A_{1} \gamma+A_{2} \tau\right)\right]}{A_{1} \gamma+A_{2} \tau},
\end{aligned}
$$

where $A_{1}\left(T_{c}, g_{c}\right), A_{2}\left(T_{c}, g_{c}\right)$ are determined in such a way that $\frac{\mathrm{d} g_{c}(T, \epsilon)}{\mathrm{d} T}=-\frac{A_{2}}{A_{1}} \frac{g_{c}}{T_{c}}, \gamma=\left(g-g_{c}\right) / g_{c}, \tau=\left(T-T_{c}\right) / T_{c}$, and $\phi_{1}=\phi_{2}=1$ for the two crossover exponents, consistently with the fact that the unzipping transition is first-order (the explicit form of $A_{1}\left(T_{c}, g_{c}\right), A_{2}\left(T_{c}, g_{c}\right)$ is worked out in Appendix B). Note that $A_{1}>0$, whereas $A_{2}$ is negative in the re-entrant part of the transition curve. The scaling functions $h_{1,2}(x)$ behave in a similar way to $f(x)$, and thus we get that $\left\langle n>\sim N^{\phi_{1}}\right.$ and $\left\langle x_{q}>\sim N^{\phi_{2}}\right.$ are both extensive at the transition point. The physical interpretation is that a macroscopic portion of the chain is still in the double stranded state, but the rest of the chain is unzipped; these are just the two phases coexisting at the first order transition. This result will be used later to justify the use of Y-shaped configurations.

It is instructive to compare this phase diagram with that of the same $D=2$-case when we allow crossing. The transition line (plotted in Fig. 2), obtained from $z_{2}=z_{3}$ (see Appendix A) is:

$$
g_{c}(T, \epsilon)=T \tanh ^{-1}[1-\exp (-\beta \epsilon)]=\frac{\epsilon}{2}+\frac{1}{2 \beta} \log [2-\exp (-\beta \epsilon)]
$$

Both the models behave similarly near $T=0$, namely they yield a transition to the cold denaturated state described in [19]. When we move at a constant force $g$ such that $g_{c}(0)<g<\operatorname{Max}_{T}\left[g_{c}(T, \epsilon)\right]$, at low enough temperatures the molecule is unzipped, and it zips by increasing $T$. This feature of the phase diagrams seems at first sight paradoxical, since one would expect the critical force to decrease monotonically as the temperature is increased. The physical explanation of this result will be given below. In the model w.c., moreover, $g_{c}$ always increases, approaching $\epsilon$ as $T \rightarrow \infty$, and the two strands remain zipped for every temperature when $g<\epsilon$. In the model w.o.c., instead, the two strands unzip again by further increasing the temperature. As regards scaling, on the other hand, the two models are identical so that we can say that the effect of forbidding crossing is irrelevant in the renormalization group sense, but has a dramatic effect on the form of the critical line.

Let us now discuss the results that we have obtained in higher dimensions (the critical lines for three and four dimensional models are shown in Fig. 3). Common to all models is the cold unzipping transition found in the $D=2$ case. Moreover, the scaling laws at the unzipping transition do not depend on dimensionality in the crossover exponents. On the other hand, the thermal melting has dimensionality-dependent critical exponents (see [11],17]). Consequently, the behaviour of the critical line $g_{c}(T, \epsilon)$ near the end point $T=T_{c}$ also depends on $D$. As $T \rightarrow T_{c}^{-}$ the result that we find in generic dimension is:

$$
\frac{g_{c}(T, \epsilon)}{T} \sim\left\{\begin{array}{ll}
\epsilon / T-\epsilon / T_{c}(D) & D=2,4 \\
\exp \left[-\frac{a}{\left(\epsilon / T-\epsilon / T_{c}(D)\right)}\right] & D=3 \\
{\left[\epsilon / T-\epsilon / T_{c}(D)\right]^{1 / 2}\left[\log \left(\epsilon / T-\epsilon / T_{c}(D)\right)\right]^{-1 / 2}} & D=5 \\
{\left[\epsilon / T-\epsilon / T_{c}(D)\right]^{1 / 2}} & D>5
\end{array},\right.
$$

where $a$ is a constant, and $T_{c}=\infty$ in $D=2,3$ for models w.c.

\section{PHYSICAL EXPLANATION OF RE-ENTRANCE}

\section{A. Re-entrance in lattice models}

To obtain some physical insight into our analytical results, we compute the free energy of a Y-shaped molecule of DNA with a force pulling at the extremities (see Fig. 4). In this way, we neglect all the configurations with bubbles. Such an approximation is valid at low $T$ and yields the exact result in the limit $T \rightarrow 0$. Since the configurations of the unzipped part are weighted by $\exp (\beta \vec{g} \cdot \vec{x})$, in this limit only the completely stretched configuration will contribute to the free energy for the unzipped part of the Y. In the limit of low temperature, the free energy is then: 


$$
F(m, N) \sim-(N-m)(\epsilon+T \log \mu)-2 g m
$$

where $m$ is the number of monomers in the unzipped part, $N$ is the total number of bases and $\mu$ is the connective constant of a single walk. Note that eq. (27) (with $\vec{g}=g(1,0, \ldots, 0)$ ) is valid also in the case of two self-avoiding walks (SAWs), since the connective constant of SAWs constrained to avoid the fully stretched unzipped part, is the same as for ordinary SAWs [27,29] (for a more detailed discussion on SAWs we refer to Appendix C). By minimizing with respect to $m$, we find the critical force $g_{c}(T, \epsilon)$ such that 27) is minimum for $m=0$ when $g<g_{c}(T, \epsilon)$, and for $m=N$ when $g>g_{c}(T, \epsilon)$ :

$$
g_{c}(T, \epsilon) \stackrel{T \rightarrow 0}{\sim} \frac{1}{2}(\epsilon+T \log \mu)
$$

This is indeed what we have found in our calculations, and confirms that re-entrance is a robust feature of lattice models, not depending on dimensionality or self-avoidance. In other words, (28) means that, at low $T$, it is more difficult to open a dsDNA helix as $T$ is increased, because the energy gain obtained through the unzipping is more than compensated by the entropy loss, since there is only one possible completely stretched configuration versus $\mu^{N-m}$ possibilities for the double stranded portion of the chain (we will see in the following section that the entropy loss in the continuum space exhibits a power law correction). For high enough $T$, on the other hand, also other configurations will contribute to the open portion, increasing its entropy, and the energy gain will eventually favour the unzipped state, except that in $D=2$ w.c., where the presence of bubble enhances the entropy of the native portion at any $T$, as explained below.

We have also calculated the phase diagram obtained by considering only the $\mathrm{Y}$ configuration: this amounts to putting the generating function for bubbles $B(z)=0$ in (5). As expected, the Y-approximation gives the exact behaviour (28) in the limit $T \rightarrow 0$, whereas it gets wronger and wronger as $T$ is increased (see Fig. 2 where we plot the two-dimensional case explicitly given by $g_{c}(T, \epsilon)=\frac{T}{2} \cosh ^{-1}[\exp (\beta \epsilon)-1]$ ). For the critical line near the melting point, this approximation yields $g_{c}(T, \epsilon) \sim\left(T_{c}-T\right)^{1 / 2}$ for all $D$, which from (26) is correct only for $D>5(d>4)$. This is because such an approach neglects bubbles, which are expected to be more and more relevant as $d$ decreases (for $d>d_{c}=4$ once the two walks have splitted away, they basically never meet again) and $T$ increases. In this view, it is clear that the puzzling behaviour found in the $D=2$ model with crossing, where $g_{c}$ increases for all $T$, is due to the growing presence of bubbles: before DNA can be unzipped, we have to disentangle all the bubbles that are forming, which is harder and harder as $T \rightarrow \infty$.

\section{B. Re-entrance in the continuum}

We now prove that also discrete chains in the continuum space undergo a cold denaturation for $T \rightarrow 0$. Let us consider two $N$-monomer chains in $\mathbf{R}^{\mathbf{d}}$ with no constraint on the directedness, with a force $\vec{g}$ pulling at the extremities and with constant unitary distance between consecutive monomers. As $\beta \rightarrow \infty$, bubbles can be neglected and only Y-shaped configurations contribute to the partition sum. The partition function for a Y-configuration then reads:

$$
Z_{N}(\beta \epsilon, \beta g)=\sum_{m=0}^{N-1} Z_{N-m}^{z}(\beta \epsilon) Z_{2 m}^{u}(\beta g),
$$

where $Z_{N-m}^{z}(\beta \epsilon)=\int_{\mathbf{R}^{\mathrm{d}}} d^{d} \vec{x}_{1} \ldots d^{d} \vec{x}_{N-m-1} \delta\left(\left|\vec{x}_{1}\right|-1\right) \ldots \delta\left(\left|\vec{x}_{N-m-1}\right|-1\right) \exp ((N-m) \beta \epsilon)$ is the partition function of the zipped portion of the strands, and $Z_{2 m}^{u}(\beta g)=\int_{\mathbf{R}^{\mathrm{d}}} d^{d} \vec{y}_{1} \ldots d^{d} \vec{y}_{2 m} \delta\left(\left|\vec{y}_{1}\right|-1\right) \ldots \delta\left(\left|\vec{y}_{2 m}\right|-1\right) \exp \left(\beta \vec{g} \cdot \sum_{i=1}^{2 m} \vec{y}_{i}\right)$ is that of the unzipped end. Chain discreteness is crucial in ensuring the validity of eq.(29) for $\beta \rightarrow \infty$, since when one bubble has formed, the length of the stretched portion of the chain can be at most $\sum_{i=1}^{2 m-1} \vec{y}_{i}=2(m-1) \frac{\vec{g}}{\mid \vec{g}}$. The integrals in (29) can be performed in any dimensions, and the evaluation of $Z_{2 m}^{u}(\beta g)$ involves the modified Bessel function of the first kind of order $\frac{d-2}{2}$. In particular, $Z_{2 m}^{u}(\beta g) \stackrel{\beta \rightarrow \infty}{\sim}\left(\frac{2 \pi}{\beta g}\right)^{m(d-1)} \exp (2 m \beta g)$, and there is a power-law correction with respect to the lattice result. We find that $Z_{N}(\beta \epsilon, \beta g) \stackrel{\beta \rightarrow \infty}{\sim} \sum_{m=0}^{N-1} \Omega_{d}^{N-m-1} \exp ((N-m) \epsilon) Z_{2 m}^{u}(\beta g)$, where $\Omega_{d}$ is the surface area of the unit sphere in $d$ dimensions. From this, the critical force is easily found:

$$
g_{c}(T, \epsilon) \stackrel{T \rightarrow 0}{\sim} \frac{\epsilon}{2}-\frac{d-1}{2} T \log \frac{T}{\epsilon}-\frac{T}{2} \log \left[2^{2 d-3} \pi^{\frac{d}{2}-1} \Gamma\left(\frac{d}{2}\right)\right],
$$

where $\Gamma$ is the Euler Gamma function. The critical line $g_{c}(T, \epsilon)$ increases at low $T$ and re-entrance is present also in the continuum, even enhanced with respect to the lattice case. The leading term $T \log T$ in equation (30) (due to the 
power-law correction in $\left.Z_{2 m}^{u}(\beta g)\right)$ is indeed not present in the $d=1$ case, when one correctly recovers equation (28) for a lattice random walk with $\mu=2$.

We finally wish to discuss the relation of the present treatment with previous work ( 13 16 $)$ done on the unzipping of homo-DNA in the limit of a continuum chain. The ds molecule with the pulling force had been described by means of an effective hamiltonian, which, apart from an irrelevant center of mass term, reads:

$$
H=\int_{0}^{N} d n\left(\frac{T d}{b^{2}}\left(\frac{d \vec{r}}{d n}\right)^{2}+V(\vec{r}(n))-\vec{g} \cdot \frac{d \vec{r}}{d n}\right),
$$

where $\vec{r}$ is the relative separation between the strands, $b$ is the effective Kuhn length of single-stranded DNA and $V$ is a realistic short range attractive potential, namely a delta function or a potential well (the two cases should be equivalent according to the standard quantum theory as long as $\int d \vec{r} V(\vec{r})$ is the same). The system described by (31) is equivalently represented by a quantum system with a non-hermitian hamiltonian. One finds 14.15] that there is a first order unzipping transition when the force reaches the critical value:

$$
g_{c}(T, \epsilon)=\sqrt{-\frac{4 \epsilon_{0}(T) T d}{b^{2}}}
$$

where $\epsilon_{0}(T)$ is the ground state energy of the quantum hamiltonian obtained from (31) when $\vec{g}=\overrightarrow{0}$. In the quantum mechanical system, for $g<g_{c}(T, \epsilon)$ the ground state is a bound state, while for $g>g_{c}(T, \epsilon)$ the spectrum of (31) is continuous.

Let us focus on the $d=1$ case, corresponding to our two-dimensional models without crossing constraints. It is well known that at $g=0$ both a symmetric square well and a delta function always have at least one bound state, meaning that DNA remains ds at all $T$. In Fig. 5 we show the critical force for the two potentials (obtained simply from (32). It can be seen from the figure that at high $T$ both the potentials saturate towards the same limit, as in our calculation for the model w.c. This is expected since the delta potential case can be recovered from our equation (2) in the limit of both continuum space and time (see Fig. 4). At low $T$, however, $g_{c}(T, \epsilon) \sim\left(\frac{T}{\Delta}\right)^{1 / 2}$ for a square well of width $\Delta$, and is constant for the delta potential, so that re-entrance is present only in the former case, but with a behaviour different from that found in the lattice. This already signals that the low temperature behaviour of the solution obtained through the quantum mapping is rather unphysical. This is due to the fact that in the limit of continuum chain the chain constraint is modelled in a 'soft' way, by using a harmonic potential between consecutive beads along the chain. This interaction is usually assumed to be entropic (as in eq. (31)) and thus effectively vanishes at $T=0$. In that limit eq. (31) is describing a set of $N$ 'free' particles moving in an effective potential determined by $V$ and $g$. Consequently, the quantum mapping is expected to describe the system well except in the low temperature limit. Indeed, the re-entrance found for the square well potential is due to the unboundedness of the potential felt by the "free particles" as soon as $g \neq 0$. As $T \rightarrow 0$, fluctuations are not important, and the particles stay close to the minimum of such potential, which is $-\infty$ for a force whatever small but finite. This is enough to unzip the strands [30].

As Zhou has observed in [16], one can improve this model by placing a hard core either inside the potential well or at a finite distance from the delta (this is analogous in spirit to introducing the crossing constraint in our lattice

models). Once again, one finds that for $\mathrm{T}$ near $T_{c}$, which is now finite, both potentials predict $g_{c}(T, \epsilon) \stackrel{T \rightarrow T_{c}}{\sim}\left(T_{c}-T\right)$ as in the lattice model (see eq. (26)), whereas for low $T$ the delta shows no re-entrance and the potential well displays a $T^{1 / 2}$ behaviour. We note once again that the low temperature behavior of such 'quantum' models is an artifact, when considered as polymer chain models, due to the 'soft' enforcing of the chain constraint. In this respect, discrete chain models in the continuum space are more realistic, either with constant bond length or with harmonic springs between consecutive beads. In the first case we have indeed proved (see eq. (30)) the existence of cold mechanical denaturation. In the second case it has been shown to occur by means of numerical simulations [19].

\section{CONCLUSIONS}

To conclude, we have studied simple models for DNA mechanical unzipping induced by a pulling force. By using analytical techniques, the relevance of forbidding the crossing of the two strands and the role played by bubble formation in the denaturation process has been discussed throughout the whole force-temperature plane. The scaling properties of the system along the transition line have also been determined. The existence of a cold mechanical denaturation for a finite range of forces at low enough temperatures has been exactly proved in a general case for both lattice and continuum space models. The fundamental role of chain discreteness has been emphasized in comparison 
with related models studied by means of quantum mapping techniques. Even if we neglected many important effects, such as base pairs heterogeneity, intrinsic helicity, wrong base pairing, and different stacking energy for the natured and denatured state, it would be interesting to experimentally test this prediction stemming from such a simple model.

Furthermore, on the basis of preliminary results (analytical and numerical) we anticipate that even in the presence of quenched randomness in the contact potential our models display a re-entrant transition, so in this sense it is really a "universal" feature.

Acknowledgements: We would like to thank F. Seno and S.M. Bhattacharjee for illuminating discussions. DM also acknowledges INFM funding. This work was supported by MURST grant.

[1] K. Svoboda, S. M. Block, Annu. Rev. Biophysics. Biomol. Structure, 23, 247, (1994).

[2] A. Ashkin, Proc. Natl. Acad. Sci. USA, 94, 4853, (1997).

[3] A. Kishino, T. Yanagida, Nature, 34, 74, (1988).

[4] H. G. Hansma, J. Vac. Sci. Technol., B14, 1390, (1995).

[5] U. Bockelmann, B. Essevaz-Roulet, F. Heslot, Phys. Rev. Lett., 79, 4489, (1997).

[6] B. Essevaz-Roulet, U. Bockelmann, F. Heslot, Proc. Natl. Acad. Sci. USA, 94, 11935, (1997).

[7] H. Yin, M. D. Wang, K. Svoboda, R. Landick, S. M. Block. J. Gelles, Science, 270, 1653, (1995).

[8] A. Kornberg, T. Baker, DNA replication, Freeman, New York, 1991.

[9] R. M. Wartell, A. S. Benight, Phys. Rep., 126, 67, (1985).

[10] B. H. Zimm, J. K. Bragg, J. Chem. Phys., 31, 526, (1959).

[11] D. Poland, H. A. Sheraga, J. Chem. Phys., 45, 1456,1464, (1966).

[12] M. Peyrard, A. R. Bishop, Phys. Rev. Lett., 62, 2755, (1989)

[13] S. M. Bhattacharjee, J. Phys. A, 33, L423 (2000); 33, 9003(E) (2000); cond-mat/0010132, (2000).

[14] D. K. Lubensky, D. R. Nelson, Phys. Rev. Lett., 85, 1572, (2000).

[15] K. L. Sebastian, Phys. Rev. E., 62, 1128, (2000).

[16] H. Zhou, cond-mat/0007015, (2000).

[17] M. S. Causo, B. Coluzzi, P. Grassberger, , Phys. Rev. E, 62, 3958, (2000).

[18] Y. Kafri, D. Mukamel, L. Peliti, Phys. Rev. Lett., 85, 4988, (2000).

[19] A. Maritan, E. Orlandini, F. Seno, unpublished; D. Marenduzzo, S. M. Bhattacharjee, A. Maritan, E. Orlandini, F. Seno, cond-mat/0103142.

[20] R. J. Rubin, J. Chem. Phys., 43, 2392, (1965).

[21] G. Forgacs et al, in Phase Transitions and Critical Phenomena, Vol.14, Edited by C. Domb, J. L. Lebowitz. Vol. I, Clarendon Press, Oxford, 1995.

[22] Note, however, that this is also the case for generic DSAWs, at least in the non-interacting case , where the walks are directed along a generic vector and the self-avoidance constraint is truly effective in the D-1-dimensional transverse space. See J. Cardy, J. Phys. A, 16, L355, (1983).

[23] N. Theodorakopoulos, T. Dauxois, M. Peyrard, Phys. Rev. Lett., 85, 6, (2000).

[24] D. Cule, T. Hwa, Phys. Rev. Lett., 79, 2375, (1997).

[25] R. J. Rubin, J. Chem. Phys., 44, 2130, (1966).

[26] S. Lifson, J. Chem. Phys., 40, 3705, (1964).

[27] B. D. Hughes, Random Walks and Random Environments, Vol. I, Clarendon Press, Oxford, 1995.

[28] For simple RWs with a force pulling in the direction $(1,0, \ldots, 0)$, the new singularity $z_{3}$ coming from the external force can be obtained in the same way as done in Section II. Its value for $d$-dimensional RWs is $\frac{1}{2(d-1+\cosh (\beta g))}$. The value of $z_{2}$ can be found as in 17 .

[29] J. M. Hammersley, G. M. Torrie, and S. G. Whittington, J. Phys. A: Math. Gen., 15, 539, (1982).

[30] The exponent $1 / 2$ is given by mean field theory: see 15.

\section{APPENDIX A: COMPUTATION OF $B(Z)$ IN MODELS OF DSAWS WITHOUT CROSSING AND OF THE PHASE DIAGRAM FOR MODELS WITH CROSSING}

We here first work out the details to obtain an explicit expression for $B(z)$, defined in eq.(6). Starting from equations (7), we can perform a Fourier and a discrete Laplace transform to obtain: 


$$
\tilde{c}(z, \vec{q}) \equiv \sum_{N=0}^{\infty} z^{N} \sum_{\vec{x}} \exp (i \vec{q} \cdot \vec{x}) c_{N}(\vec{x})=\frac{1}{1-z f(\vec{q})}\left(\tilde{c_{0}}(\vec{q})-z f_{0}(\vec{q}) c(z, \overrightarrow{0})\right)
$$

where we have made the following definitions:

$$
\begin{aligned}
& f_{0}(\vec{q}) \equiv 2 \sum_{i<j ; i, j=1}^{D} \cos \left(q_{i}-q_{j}\right) ; f(\vec{q}) \equiv D+f_{0}(\vec{q}) \\
& c(z, \vec{x}) \equiv \sum_{N=0}^{\infty} z^{N} c_{N}(\vec{x}) ; \quad \widetilde{c_{0}}(\vec{q}) \equiv \sum_{\vec{x}} \exp (i \vec{q} \cdot \vec{x}) c_{0}(\vec{x})
\end{aligned}
$$

Using $c(z, \overrightarrow{0})=\int_{[-\pi, \pi]^{D}} \frac{d^{D} \vec{q}}{(2 \pi)^{D}} \widetilde{c}(z, \vec{q})$, equation (A1) is easily solved since, by permuting variables inside the resulting integrals, it is possible to see that they do not depend on the particular step which we forbid. We obtain:

$$
\begin{aligned}
\tilde{c}(z, \vec{q}) & =\frac{1}{1-z f(\vec{q})} \frac{\tilde{c_{0}}(\vec{q})-z W_{1}(z)\left[A(D) f_{0}(\vec{q})-\tilde{c_{0}}(\vec{q})\right]}{1+z W_{1}(z)}, \\
c(z, \overrightarrow{0}) & =A(D) \frac{W_{1}(z)}{1+z W_{1}(z)},
\end{aligned}
$$

where $A(D)=1-\frac{1}{D(D-1)}$ is the reduction factor due to the crossing constraint. As a result, using $B(z)=$ $z^{2} \sum_{i \neq j ; i, j=1}^{D} c\left(z, \vec{e}_{i}-\vec{e}_{j}\right)$, we obtain

$$
B(z)=A(D) \frac{z^{2} W_{2}(z)}{1+z_{2} W_{1}(z)}
$$

where

$$
W_{1}(z)=\int_{[-\pi, \pi]^{D}} \frac{d^{D} \vec{q}}{(2 \pi)^{D}} \frac{f_{0}(\vec{q})}{1-z f(\vec{q})} \quad, \quad W_{2}(z)=\int_{[-\pi, \pi]^{D}} \frac{d^{D} \vec{q}}{(2 \pi)^{D}} \frac{f_{0}^{2}(\vec{q})}{1-z f(\vec{q})}
$$

The singularity $z_{1}=1 / D^{2}$, leading to the usual random walk behavior, comes in when evaluating the above integrals; their denominator becomes negative as $\vec{q} \rightarrow 0$ for $z>z_{1}$.

We give here also a brief outline of the calculation necessary to find out the phase diagram for the models of Section II of DSAWs when crossing is allowed. These models are simpler to solve than the corresponding models w.o.c., since it is not necessary to partition the molecule of DNA as done in the text in eq.(5), which allows us to write a more explicit formula for the grand partition function. Indeed we can simply start from the recursion relations (2) and, after Fourier and Laplace transform, find the expression:

$$
\tilde{p}(z, \vec{q}, \beta \epsilon)=\frac{1}{1-z f(\vec{q})} \frac{1}{1-(1-\exp (-\beta \epsilon)) W_{0}(z)},
$$

for the quantity $\tilde{p}(z, \vec{q}, \beta \epsilon)$, defined as:

$$
\tilde{p}(z, \vec{q}, \beta \epsilon)=\sum_{N=0}^{\infty} z^{N} \sum_{\vec{x}} \exp (i \vec{q} \cdot \vec{x}) p_{N}(\vec{x}, \beta \epsilon)
$$

with $p_{N}(\vec{x}, \beta \epsilon)$ defined as in eq.(1) and

$$
W_{0}=\int_{[-\pi, \pi]^{D}} \frac{d^{D} \vec{q}}{(2 \pi)^{D}} \frac{1}{1-z f(\vec{q})} .
$$

The final form of the grand partition function, for DSAWs w.c. in the presence of a pulling force, is:

$$
Z(z, \beta \epsilon, \beta g)=\frac{1}{1-z / z_{3}(\beta g)} \frac{1}{1-(1-\exp (-\beta \epsilon)) W_{0}(z)},
$$

with $z_{3}(\beta g)$ defined as in eq.(15). Alternatively, one can proceed exactly as in Section II, but with initial conditions 


$$
c_{0}(\vec{x})=\sum_{i \neq j ; i, j=1}^{D} \delta_{\vec{x}, \vec{e}_{i}-\vec{e}_{j}} .
$$

The only resulting difference would be to have $A(D)=1$ in eqs. (A4) and (A6); after a bit of algebra one can convince himself that the partition function found by recollecting the different factors in eq.(5) is the same as that found in eq. A11). In doing this, one needs to compute the relations between the integrals $W_{0}(z), W_{1}(z), W_{2}(z)$, which may be done as, e.g., in 25]. For example, the bubble generating function for both kind of models could also be simply expressed as

$$
B(z)=A(D)\left(1-D z-1 / W_{0}(z)\right)
$$

Changing the dimension dependent factor $A(D)$ from $A(D)=1$, when crossing is allowed, to $A(D)=1-\frac{1}{D(D-1)}$, when crossing is forbidden, is enough for the melting transition temperature to become finite for $D \leq 3$.

\section{APPENDIX B: TAUBERIAN THEOREM AND ITS APPLICATIONS}

The discrete Tauberian theorem (see [27]) states that the relations

$$
c(z) \equiv \sum_{N=0}^{\infty} c_{N} z^{N} \stackrel{z \rightarrow z^{*-}}{\sim}\left(\frac{1}{1-z / z^{*}}\right)^{\rho} L\left(\frac{1}{1-z / z^{*}}\right)
$$

and

$$
c_{N} \stackrel{N \rightarrow \infty}{\sim} \frac{N^{\rho-1}}{\left(z^{*}\right)^{N} \Gamma(\rho)} L(N)
$$

are equivalent provided that $\rho>0,\left\{c_{N}\right\}$ is a positive monotonic sequence and $L$ is "slowly varying" in the sense specified in [27].

We apply this theorem here first to find the scaling laws of the unzipping transition to give an example of how the relations reported in section III can be recovered. When approaching the critical line at $g=g_{c}$ from the unzipped phase, the smallest singularity is $z_{3}(\beta g)$, as defined in eq. (15). The leading behavior as $z \rightarrow z_{3}^{-}$is:

$$
\begin{aligned}
Z(z, \beta \epsilon, \beta g) & \stackrel{z \rightarrow z_{3}^{-}}{\sim} \frac{1}{\exp (-\beta \epsilon)-D z_{3}-B\left(z_{3}\right)} \frac{1}{1-z / z_{3}}, \\
\frac{\partial Z(z, \beta \epsilon, \beta g)}{\partial(\beta g)} & \stackrel{z \rightarrow z^{-}}{\sim}-\frac{1}{\exp (-\beta \epsilon)-D z_{3}-B\left(z_{3}\right)} \frac{1}{\left(1-z / z_{3}\right)^{2}} \frac{\partial z_{3} / \partial(\beta g)}{z_{3}(\beta g)}, \\
\frac{\partial Z(z, \beta \epsilon, \beta g)}{\partial(\beta \epsilon)} & \stackrel{z \rightarrow z^{-}}{\sim} \frac{\exp (-\beta \epsilon)}{\left(\exp (-\beta \epsilon)-D z_{3}-B\left(z_{3}\right)\right)^{2}} \frac{1}{1-z / z_{3}},
\end{aligned}
$$

where we have neglected inessential factors when $g \sim g_{c}$. Consequently, the application of the Tauberian theorem yields:

$$
\begin{aligned}
Z_{N}(\beta \epsilon, \beta g) & \stackrel{N \rightarrow \infty}{\sim} \frac{1}{\exp (-\beta \epsilon)-D z_{3}-B\left(z_{3}\right)} z_{3}^{-N}, \\
\frac{\partial Z_{N}(\beta \epsilon, \beta g)}{\partial(\beta g)} & \stackrel{N \rightarrow \infty}{\sim}-\frac{N}{\exp (-\beta \epsilon)-D z_{3}-B\left(z_{3}\right)} z_{3}^{-N} \frac{\partial z_{3} / \partial(\beta g)}{z_{3}(\beta g)}, \\
\frac{\partial Z_{N}(\beta \epsilon, \beta g)}{\partial(\beta \epsilon)} & \stackrel{N \rightarrow \infty}{\sim} \frac{\exp (-\beta \epsilon)}{\left(\exp (-\beta \epsilon)-D z_{3}-B\left(z_{3}\right)\right)^{2}} z_{3}^{-N} .
\end{aligned}
$$

The canonical partition function $Z_{N}$ can be expressed in terms of previously defined quantities as $Z_{N}(\beta \epsilon, \beta g)=$ $\sum_{\vec{x}} p_{N}(\vec{x}, \beta \epsilon) \exp (\beta \vec{g} \cdot \vec{x})$ (see eqs.(1) and (位).

Note that at the critical line $z_{2}(\beta \epsilon)=z_{3}\left(\beta g_{c}\right)$, implying that the $\epsilon$-dependent denominator in the above equations vanishes, as the critical line is approached (that is $\gamma \equiv\left(g-g_{c}\right) / g_{c} \ll 1$ and $\left.\tau \equiv\left(T-T_{c}\right) / T_{c} \ll 1\right)$. In that limit the order parameters obey 


$$
\begin{aligned}
& <n>\equiv \frac{\partial Z_{N} / \partial(\beta \epsilon)}{Z_{N}} \stackrel{N \rightarrow \infty}{\sim} \frac{\exp (-\beta \epsilon)}{A_{1} \gamma+A_{2} \tau}, \\
& <x_{g}>\equiv-\frac{\partial Z_{N} / \partial(\beta g)}{Z_{N}} \stackrel{N \rightarrow \infty}{\sim} \frac{\partial z_{3} / \partial(\beta g)}{z_{3}\left(\beta g_{c}\right)} N .
\end{aligned}
$$

The coefficients $A_{1}\left(T_{c}, g_{c}\right), A_{2}\left(T_{c}, g_{c}\right)$ determine the normal direction in the $(\log (T), \log (g))$ plane, and are reported below:

$$
\begin{aligned}
A_{1}\left(T_{c}, g_{c}\right)= & \beta_{c} g_{c}\left(D+\frac{\partial B\left(z_{3}\left(\beta_{c} g_{c}\right)\right)}{\partial z_{3}}\right) z_{3}^{2}\left(\beta_{c} g_{c}\right)\left[4 \sinh \left(2 \beta_{c} g_{c}\right)+4(D-2) \sinh \left(\beta_{c} g_{c}\right)\right], \\
A_{2}\left(T_{c}, g_{c}\right)= & -\beta_{c} g_{c}\left(D+\frac{\partial B\left(z_{3}\left(\beta_{c} g_{c}\right)\right)}{\partial z_{3}}\right) z_{3}^{2}\left(\beta_{c} g_{c}\right)\left[4 \sinh \left(2 \beta_{c} g_{c}\right)+4(D-2) \sinh \left(\beta_{c} g_{c}\right)\right]+ \\
& \beta_{c} \epsilon \exp \left(-\beta_{c} \epsilon\right) .
\end{aligned}
$$

As expected, it may be easily checked that, whatever direction we choose to approach the critical curve, the physical quantities as (B9) only depend on the projection of this direction on the normal to the critical line. To show this, one only needs first to observe that criticality is achieved through the vanishing of the denominator in (Bי), and then to apply the implicit function theorem to this denominator.

Recently, some authors ( 13, 14 ) have suggested, as an alternative order parameter for the unzipping transition, the number $m$ of monomers from the last contact to the end. By using the same tools as before, we can find some quantities of interest such as the probability distribution of having $m$ monomers "liberated" (in the equations below we suppose $1<<m<<\mathrm{N})$. In general:

$$
P(m)=\frac{\sum_{W} \delta_{n_{u, z}, m} p_{W}}{\sum_{W} p_{W}}
$$

where $\mathrm{W}$ are the pairs of directed walks, $p_{W}$ is their Boltzmann weight and $n_{u . z}$. is the number of "liberated" monomers of the configuration. The possible enforcing of the crossing constraint does not affect the following equations, simply shifting the critical melting temperature $T_{c}$.

At zero force and $T<T_{c}$, in the native state:

$$
P(m) \propto \exp \left[-m \log \left(z_{1} / z_{2}\right)\right] m^{\alpha(d)},
$$

where $m^{\alpha(d)}=m^{-1 / 2}$ in $d=1,(\log (m))^{-1}$ in $d=2$ and constant for $d>2$. We recall that $z_{1}=1 / D^{2}$, with $D=d+1$. At criticality:

$$
P(m) \propto(N-m)^{\phi_{t}-1} m^{\alpha(d)},
$$

where in the first factor there are logarithmic corrections in $d=2,4$ to the power-law behavior controlled by the crossover thermal exponent $\phi_{t}[17]$. Above $T_{c}$ we have:

$$
P(m) \propto(N-m)^{\beta(d)} m^{\alpha(d)} .
$$

with $\beta(d)=-|1-d / 2|-1$ if $d \neq 2$, in $d=2 m^{\beta(d)}=1 /\left(N \log ^{2}(N)\right)$. At force different from zero, in the zipped phase, we get:

$$
P(m) \propto \exp \left(-m \log \left(z_{3} / z_{2}\right)\right)
$$

while in the unzipped phase:

$$
P(m) \propto \exp \left(-(N-m) \log \left(z_{2} / z_{3}\right)\right),
$$

if $T<T_{c}$, and

$$
P(m) \propto \exp \left(-(N-m) \log \left(z_{1} / z_{3}\right)\right),
$$

if $T>T_{c}$. Just at criticality, the probability distribution is flat.

For the Y-shaped configuration, we get, at force different from zero: 


$$
\begin{array}{ll}
P(m) \propto \exp \left(-(N-m) \log \left(z_{y} / z_{3}\right)\right) & \text { u.z. phase, } \\
P(m) \propto \exp \left(-m \log \left(z_{3} / z_{y}\right)\right) & \text { z. phase, } \\
P(m) \propto \text { const. } & \text { at criticality, }
\end{array}
$$

where $z_{y}=\exp (-\beta \epsilon) / \mu$ is the singularity controlling the free energy per monomer in the Y-zipped phase, and $\mu=D$ the connective constant of a single directed walk. At zero force we get:

$$
\begin{array}{ll}
P(m) \propto \exp \left(-(N-m) \log \left(z_{y} / z_{1}\right)\right) m^{\alpha(d)} & \text { denatured phase, } \\
P(m) \propto \exp \left(-m \log \left(z_{1} / z_{y}\right)\right) m^{\alpha(d)} & \text { native phase, } \\
P(m) \propto m^{\alpha(d)} & \text { at criticality. }
\end{array}
$$

Let us give the details in a simple case, to get e.g. eqns.(B20, B21, B22). One has:

$$
P(m)=\frac{Z_{N-m}^{P S} Z_{m}^{\text {free }}}{Z_{N}}
$$

where $Z_{N-m}^{P S}$ is the partition function of two $(N-m)$-monomer chains with the end points in common (this is the original Poland-Sheraga model), $Z_{m}^{\text {free }}$ is the partition of two $m$-monomer chains with no base pair in contact, and $Z_{N}$ is given by eq.(11). We have, for the $\mathrm{Y}$ at non zero force, $Z_{N-m}^{P S}=z_{y}^{-(N-m)}, Z_{m}^{\text {free }} \sim z_{3}^{-m}$, and

$$
\begin{aligned}
& Z_{N} \sim z_{y}^{-N} \quad \text { native phase, } \\
& Z_{N} \sim z_{3}^{-N} \quad \text { above criticality. }
\end{aligned}
$$

\section{APPENDIX C: Y CONFIGURATIONS WITH SAWS}

In this Appendix we extend the analysis of section IV to a $\mathrm{Y}$ configuration where the two chains are generic SAWs and not directed walks. We take here $\vec{g}=g(1,0, \ldots, 0)$.

Let us call $c_{N}(\overrightarrow{0}, \vec{x})$ the number of $N$-step SAWs ending in $\vec{x}$ and starting in $\overrightarrow{0}$, and $c_{N, \vec{g}}=\sum_{\vec{x}} c_{N}(\overrightarrow{0}, \vec{x}) \exp (\beta \vec{g} \cdot \vec{x})$ (so that $c_{N} \equiv c_{N, \vec{g}=\overrightarrow{0}}$ ). As a first step, we need to prove the existence of a connective constant $\mu_{\vec{g}}$ for a single SAW in the presence of a pulling force i.e. $c_{N, \vec{g}} \sim \mu_{\vec{g}}^{N}$ for large $N$. The existence of a connective constant then follows [27] from the inequality:

$$
\sum_{\vec{x}} c_{N}(\overrightarrow{0}, \vec{x}) \exp (\beta \vec{g} \cdot \vec{x}) \leq \sum_{\vec{x}, \vec{x}_{1}} c_{N_{1}}\left(\overrightarrow{0}, \vec{x}_{1}\right) c_{N-N_{1}}\left(\vec{x}_{1}, \vec{x}\right) \exp \left(\beta \vec{g} \cdot \vec{x}_{1}\right) \exp \left(\beta \vec{g} \cdot\left(\vec{x}-\vec{x}_{1}\right)\right),
$$

which implies the subadditivity of $c_{N}$ [27]. From the connective constants of RWs and DSAWs, we can estabilish the following bounds (recall that here $\vec{g}=g(1,0, \ldots, 0)$ ) for the connective constant of a SAW in $d$-dimensions:

$$
d-1+\exp (\beta|\vec{g}|) \leq \mu_{\vec{g}} \leq 2(d-1)+2 \cosh (\beta|\vec{g}|) .
$$

Let us now introduce the canonical partition function. It reads:

$$
Z_{N}=\sum_{m=0}^{N-1} \exp (\beta(N-m) \epsilon) c_{N-m-1} \sum_{\vec{x}_{1}, \vec{x}_{2}} c_{2 m}^{\prime}\left(\vec{x}_{1}, \vec{x}_{2}\right) \exp \left(\beta \vec{g} \cdot\left(\vec{x}_{2}-\vec{x}_{1}\right)\right),
$$

where $c_{2 m}^{\prime}\left(\vec{x}_{1}, \vec{x}_{2}\right)$ counts the SAWs which do not cross the first $N-m$ attached monomers of the $\mathrm{Y}$ and $\vec{x}_{1}, \vec{x}_{2}$ are the end-points of the strands.

An upper bound is found if we allow the opened part of the Y to cross the first $N-m$ zipped monomers:

$$
Z_{N}^{\text {upper }}=\sum_{m=0}^{N-1} \exp (\beta(N-m) \epsilon) c_{N-m-1} \sum_{\vec{x}_{1}, \vec{x}_{2}} c_{2 m}\left(\vec{x}_{1}, \vec{x}_{2}\right) \exp \left(\beta \vec{g} \cdot\left(\vec{x}_{2}-\vec{x}_{1}\right)\right) .
$$

The grand canonical partition function defined from (C4) displays singularities in $z \equiv z_{1}=\frac{1}{\mu_{\vec{g}}^{2}}$ and in $z \equiv z_{2}=$ $\frac{1}{\mu \exp (\beta \epsilon)}$, where $\mu$ is the connective constant of a standard SAW in $d$-dimensional space, i.e. $\mu=\mu_{\vec{g}=\overrightarrow{0}}$. 
To find a lower bound for $Z_{N}$, we restrict the $\mathrm{Y}$ configurations to those with the joined part of the $\mathrm{Y}$ constrained to lay in the half-space $\left\{\vec{x} \mid \vec{x} \cdot \vec{e}_{1}>0, \vec{e}_{1} \cdot \frac{\vec{g}}{|\vec{g}|}=0\right\}$ (the origin is in the bifurcation point), and the unzipped part forced to stay in the other half-space.

$$
Z_{N}^{\text {lower }}=\sum_{m=0}^{N-1} \exp (\beta(N-m) \epsilon) c_{N-m-1}^{\text {left }} \sum_{\vec{x}_{1}, \vec{x}_{2}} c_{2 m}^{r i g h t}\left(\vec{x}_{1}, \vec{x}_{2}\right) \exp \left(\beta \vec{g} \cdot\left(\vec{x}_{2}-\vec{x}_{1}\right)\right),
$$

where we have indicated with $c_{n}^{\text {left(right) }}$ the number of $n$-step SAWs constrained to stay in the left(right) half space. As the connective constants can be rigorously proved to be the same even for walks constrained in such a way (see [27]29] for details, it is important here that walks generated from a reflection through the hyperplane $\left\{\vec{x} \mid \vec{x} \cdot \vec{e}_{1}>\right.$ $\left.0, \vec{e}_{1} \cdot \frac{g}{|\vec{g}|}=0\right\}$ leave the scalar product $\vec{g} \cdot \vec{x}$ unchanged), the singularities of the grand partition function obtained from $(\overrightarrow{\mathrm{C} 5})$ are the same as those of $\sum_{N=0}^{\infty} Z_{N}^{\text {upper }} z^{N}$. Thus the grand partition function singularities associated to $Z_{N}$ in eq.(C3) are $z=z_{1}, z_{2}$ given above.

In summary, we have proved that a mechanical unzipping transition takes place when $\mu_{\vec{g}}^{2}=\mu \exp (\beta \epsilon)$, similarly to the directed walk case. We suggest that a standard computation of $\mu_{\vec{g}}$ (for instance through exact enumeration) could give an accurate phase diagram for the "Y approximation" with SAWs.

The bounds in eq. (C2) give immediately two bounds within which the transition line $g_{c}(T, \epsilon)$ lies. In the $T \rightarrow 0$ limit eq.(28) follows since both bounds give the same asymptotic transition line. This should be the same also for generic SAWs, since in the $T \rightarrow 0$ limit it is sufficient to consider only Y-shaped configurations. This demonstrates the existence of cold unzipping at sufficiently low temperature for the generic self-avoiding case. 


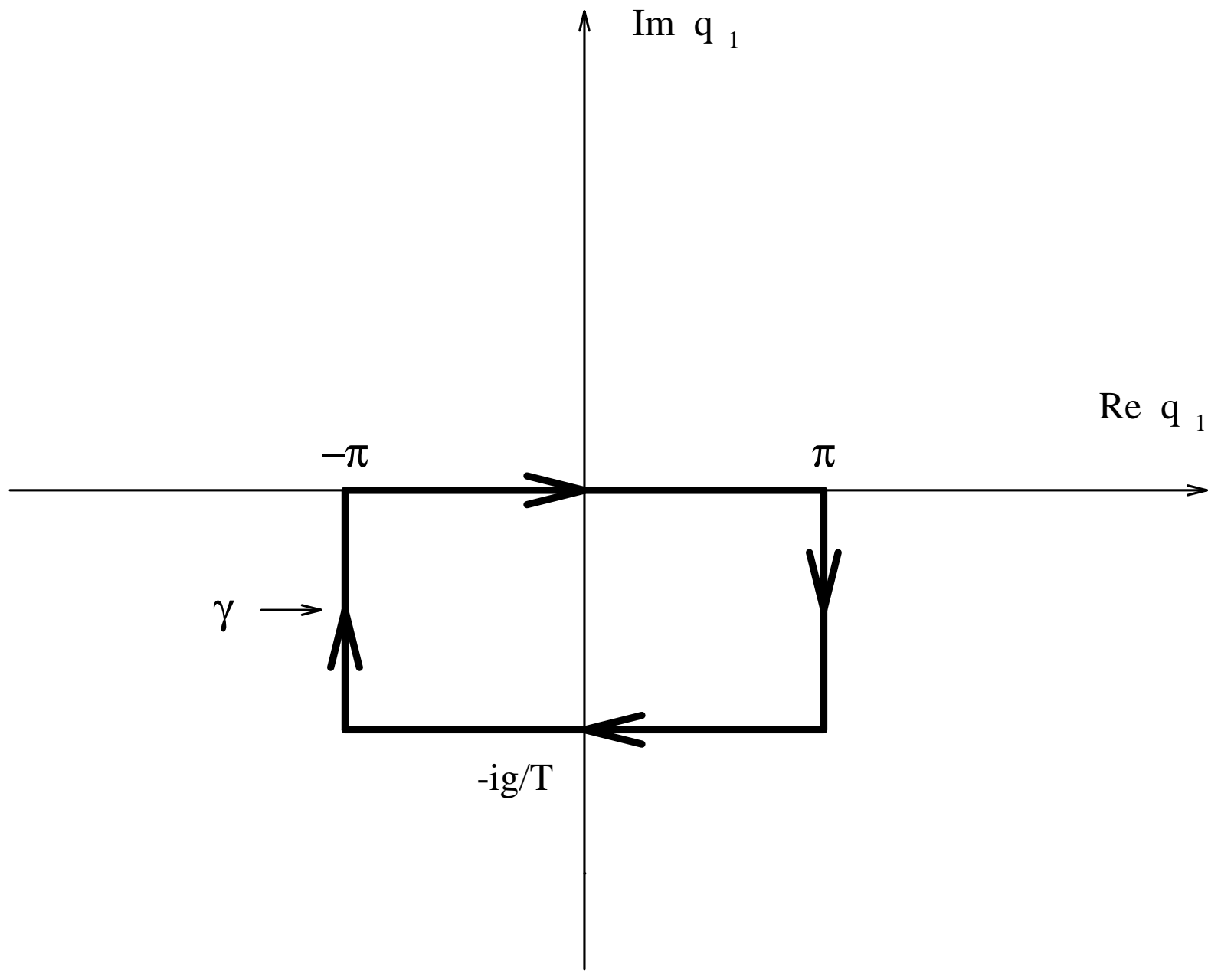

FIG. 1. Contour of integration $\gamma$ in the complex plane used for the evaluation of the integrals in (14). 


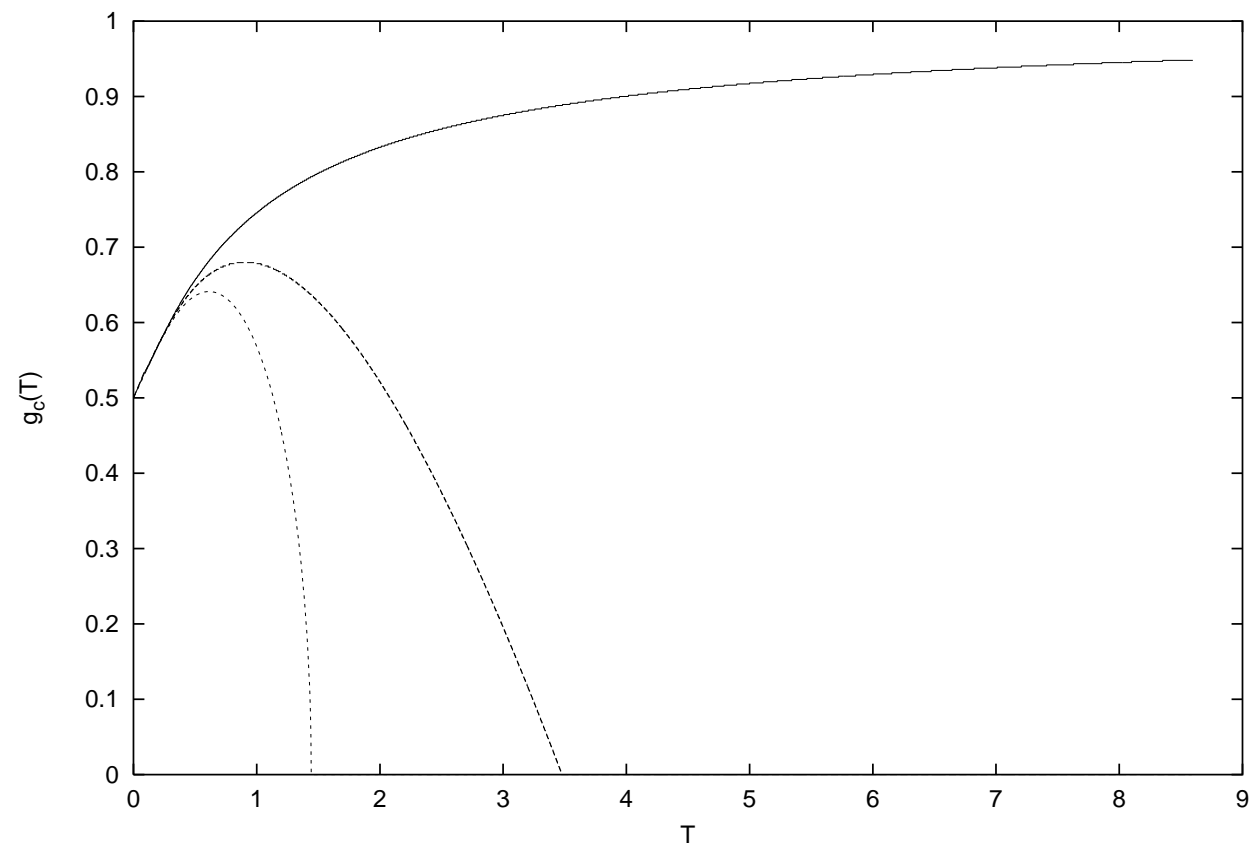

FIG. 2. Phase diagram for two-dimensional models of DSAWs $(\epsilon=1$ in the figure). The solid curve refers to the model with crossing, the long-dashed one to that without crossing and the dashed one to the model which considers only Y-shaped configurations. 


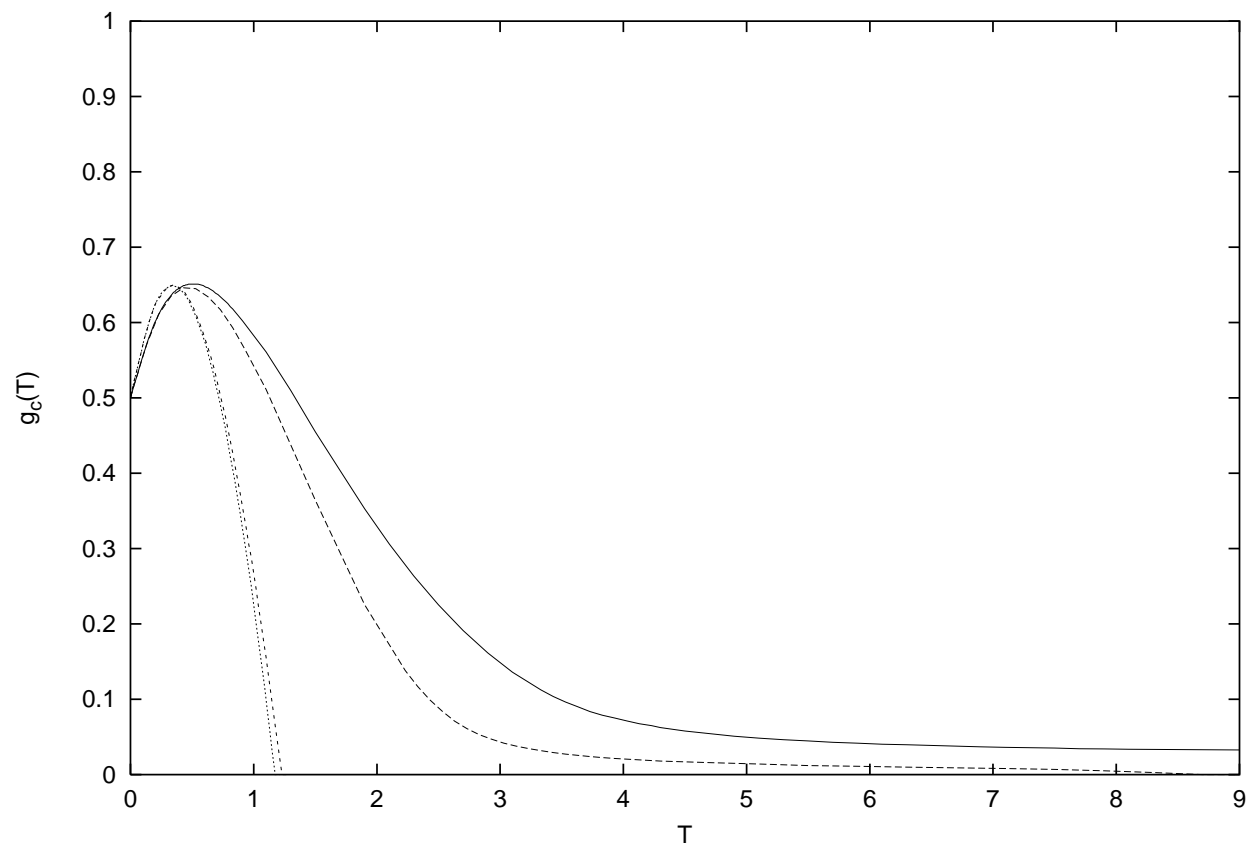

FIG. 3. Phase diagrams in $\mathrm{D}=3$ and 4 dimensions for models with and without crossing $(\epsilon=1$ in the figure). It can be seen that the difference between the four dimensional models is negligible. In $\mathrm{D}=3$ (model w.o.c.), $T_{c} \simeq 8.49 \epsilon$. Solid line: model $\mathrm{D}=3$ w.c.;

Long-dashed line: model $\mathrm{D}=3$ w.o.c.;

Dashed line: model $\mathrm{D}=4$ w.c.;

Dotted line: model $\mathrm{D}=4$ w.o.c. 
a)

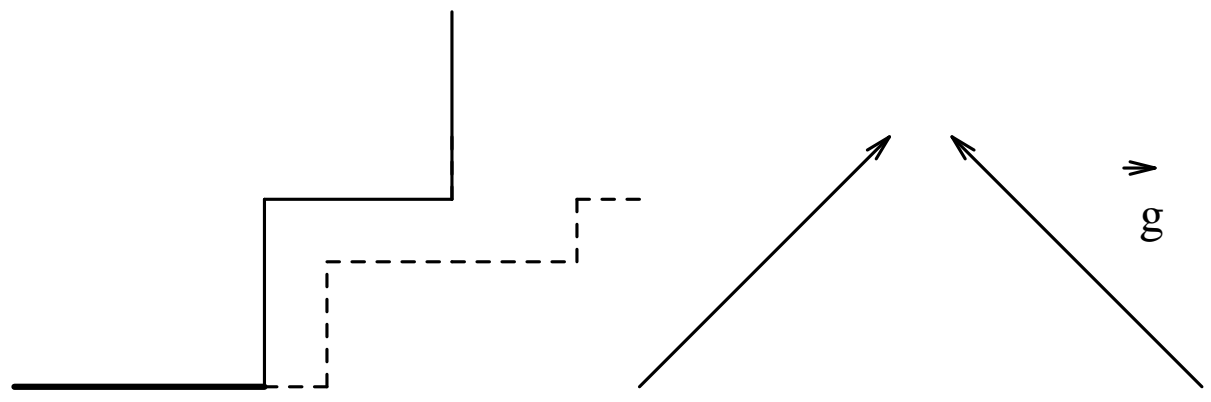

b)
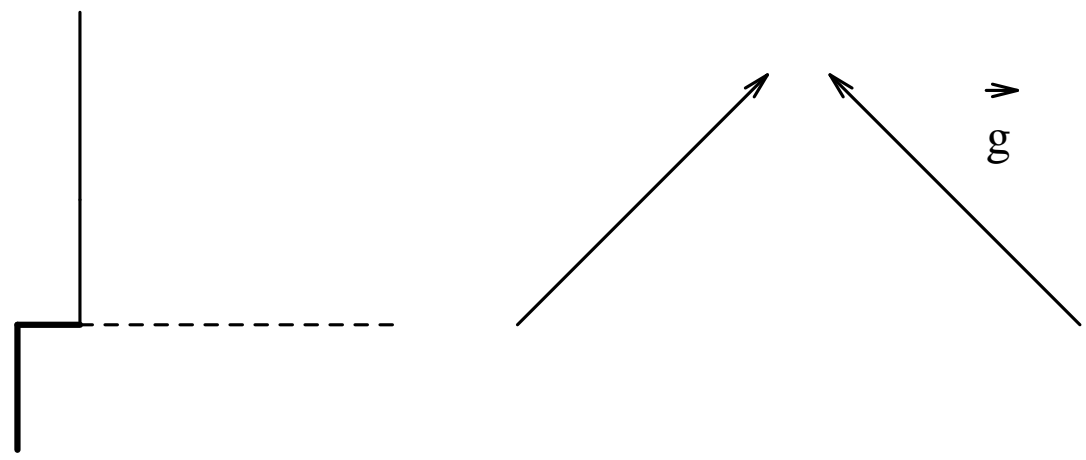

FIG. 4. a)We show here an example of Y-shaped configuration for the DNA molecule: in this approximation bubbles are neglected.

b)A completely stretched configuration for directed walks, which is dominant for $T \rightarrow 0$. 


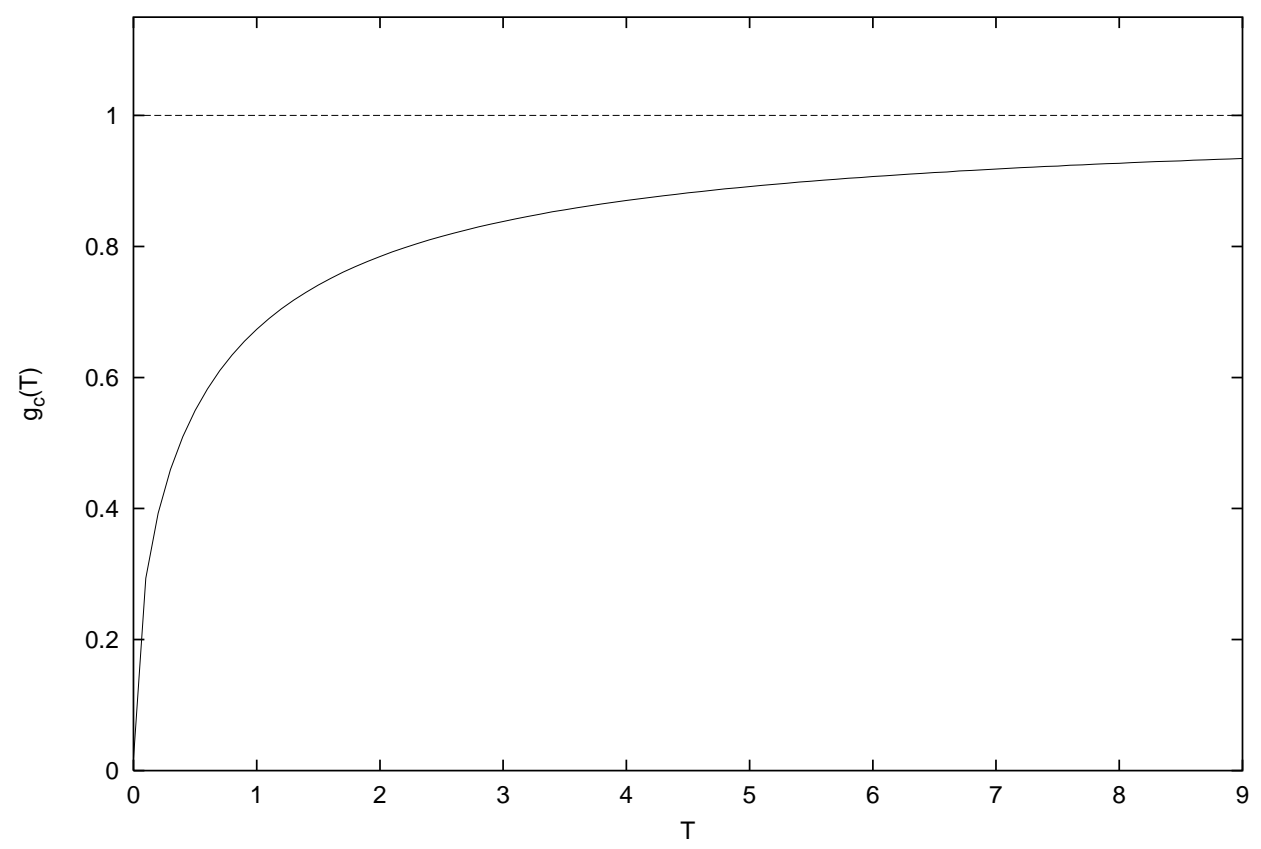

FIG. 5. Critical force in $d=1$, found with the quantum mapping. The solid line is the result with a symmetric square well, the dashed one with a delta function. The parameters are chosen so that the integral of the potentials over space is the same. 